\title{
ESTRATEGIA DE MARKETING RELACIONAL PARA LOGRAR LA FIDELIZACIÓN DE LOS CLIENTES
}

\author{
Relational marketing strategy to achieve customer loyalty \\ Universidad Peruana Unión
}

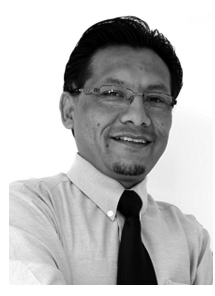

\section{Juan Carlos Niño de Guzmán Miranda}

Licenciado en Administración por la Universidad Peruana Unión. Magíster en Marketing y Negocios Internacionales por la Universidad Nacional Federico Villarreal. Actualmente se desempeña como coordinador de la Facultad de Ciencias Empresariales de la Universidad Peruana Unión - Campus Tarapoto y director de la Oficina de Planificación y Desarrollo de la misma. 


\section{Resumen}

El objetivo de esta investigación es determinar la eficacia de la estrategia de marketing relacional para mejorar la fidelización de los clientes en el Centro de Aplicación Productos Unión. La investigación tiene un diseño preexperimental. Para la recolección de información se aplicó la Encuesta de Fidelización de Clientes, en una muestra de 216 bodegueros de Lima Metropolitana, en dos momentos: antes y después de la implementación de la estrategia de marketing relacional. La encuesta mide tres dimensiones de la fidelización de los clientes: compra, servicios y lealtad. Los resultados muestran que la fidelización de los clientes se incrementó después de la implementación de la estrategia de marketing relacional, teniendo como media del pretest $=59.82$ y el postest $=93.25$. Del mismo modo, se incrementaron las medias en las dimensiones compra (pretest $=29.14$; postest $=47.70$ ), servicios (pretest $=25.96$; postest $=31.53)$, y lealtad $($ pretest $=3.78$; postest $=$ 9.49). La prueba "t de student" para el contraste de medias muestra que hubo un incremento significativo en la fidelización de los clientes (0.000), compra (0.000), servicios $(0.000)$ y lealtad $(0.000)$ a un nivel de confianza de $95 \%$. En conclusión, la estrategia de marketing relacional mejora la fidelización de los clientes.

Palabras clave: Cliente; fidelización; marketing; marketing relacional.

\section{Abstract}

This research's objective is to determine the effectiveness of relational marketing strategy to improve customer loyalty in the Centro de Aplicación Productos Unión. The research has a pre-experimental design, before and after the implementation of the relational marketing strategy: to collect information a Customer Loyalty Survey was applied on a sample of 216 small stores of Metro Lima, in two stages: before and after the implementation of the relational marketing strategy.The survey measures three dimensions of customer loyalty: purchase, service and loyalty. The results show that customer loyalty increased after the implementation of the relational marketing strategy, with averages: pretest $=59.82$ and and posttest $=93.25$. Similarly, the average increased in the purchase (pretest $=29.14$, posttest $=47.70$ ), services (pretest $=25.96$, posttest $=31.53$ ), and loyalty (pretest $=3.78$, posttest $=9.49$ ) dimensions. The "t de student" for the comparison of the means shows that there was a significant increase in customer loyalty $(0.000)$, purchase $(0.000)$, services $(0.000)$ and loyalty $(0.000)$ at a confidence level of $95 \%$. In conclusion, the relational marketing strategy improves customer loyalty.

Keywords: Customer; loyalty; marketing; relationship marketing. 


\section{Introducción}

La fidelización de los clientes, usuarios o compradores es hoy en día una de las prioridades principales de las organizaciones; la economía de la mayoría de países del orbe en la que se desarrollan los negocios tiene como centro al cliente, quien decide finalmente el destino de muchas organizaciones con su elección de compra o consumo recurrente de determinado producto o servicio. Según Alfaro (2004), la fidelidad puede definirse como una actitud favorable hacia una marca que se configura en una repetición de actos de compra. De esta forma se desarrolla el proceso de aprendizaje del cliente respecto a la capacidad de la misma marca de satisfacer sus expectativas y, por consecuencia, acumula confianza hacia la empresa. En este sentido, Reichheld (2002), sostiene que la fidelización es la única vía para alcanzar utilidades superiores sostenibles.

Al respecto, se han planteado teorías que intentan explicar y prever cómo llegar a obtener clientes fieles. Sheth, Mittal y Newman (2001), proponen que una forma de trabajar con el tema de la fidelización es considerando dos factores primordiales que son el "qué" y el "cómo". Se encontró en una investigación realizada acerca de los atributos determinantes de la lealtad bajo la óptica de dos constructos: la compra y el servicio, mediante estos se analiza tal fidelidad de clientes (Mesquita y Lara, 2007). En la revisión de trabajos previos, Acevedo (2008) demostró que la gestión de la base de clientes permite maximizar la rentabilidad de la relación y la gestión de la lealtad es un componente estratégico para la organización. Asimismo, Payne, Ballantyne y Martin (2005), demostraron que existen seis mercados (de clientes, de proveedores y alianzas, de referencias, de influencias, de reclutamiento, y el interno) de estos el mercado de clientes es el central y de la intersección de estos mercados se pueden generar diversas estrategias de retención, de relaciones de alta intensidad, de creación de valor o de marketing transaccional. Volle y Mimouni-Chaabane (2010), investigaron algunos beneficios percibidos como resultado de los programas de lealtad, hallaron cinco tipos de beneficios percibidos: ahorro monetario, exploración, entretenimiento, reconocimiento, y beneficios sociales.

El marketing relacional es una de las vertientes del marketing que en el presente se ha convertido en una estrategia eficaz para que las organizaciones puedan mantener clientes satisfechos y con la posibilidad de considerarse fieles.

Este es el motivo por el cual se hizo esta investigación, para determinar la eficacia de la estrategia de marketing relacional y mejorar la fidelización de los clientes, en este caso en el Centro de Aplicación Productos Unión de la Universidad Peruana Unión. 


\section{Materiales y métodos}

Tiene un diseño preexperimental, asimismo se harán análisis descriptivos correlacionales. Al respecto, Bernal (2010) señala que "los sujetos participantes de la investigación se pueden asignar aleatoriamente a los grupos y algunas veces se tiene grupo de control... Se puede utilizar en diseños de un grupo con medición antes y después".

El ambiente en el que se desarrolló fue en la ciudad de Lima; el proceso fue en dos etapas: una "antes" y otra "después" de la implementación de la estrategia de marketing relacional. El grupo al cual se tomó en cuenta para esta investigación está conformado por 216 bodegueros (tenderos) de Lima Metropolitana, quienes compran frecuentemente "Productos Unión" debido a la demanda que tienen de estos en sus respectivas bodegas, y fueron elegidos aleatoriamente sin distinguir edad, condición social, religión, raza, sexo ni su nivel de educación. El muestreo utilizado fue el aleatorio simple. La población total fue de 2,200 bodegueros que realizan compras de manera recurrente. Las técnicas utilizadas para esta investigación fueron: el análisis documental, la observación y las encuestas. El diseño de la investigación se puede resumir de la siguiente manera:

\section{Figura $\mathbf{N}^{\circ} 1$}

Diseño de la investigación

$$
\mathrm{G}_{\mathrm{E}}: \mathrm{O}_{1} \rightarrow \mathrm{x} \quad \rightarrow \mathrm{O}_{2}
$$

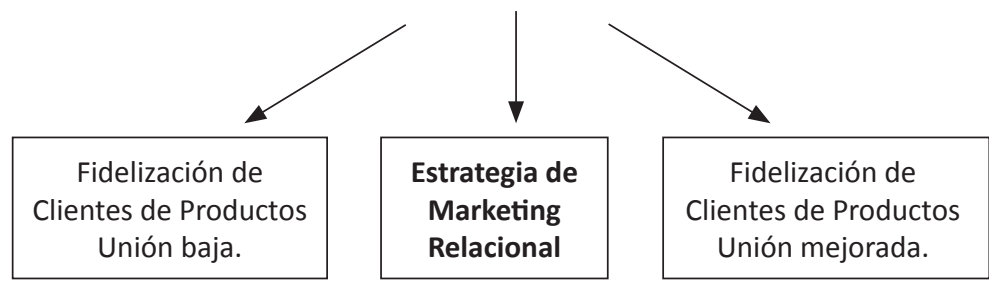

$\mathrm{G}_{\mathrm{E}}$ : Grupo Experimental - Bodegueros de Lima Metropolitana

$\mathrm{O}_{1}$ : Observación $1 \longrightarrow$ pretest (encuesta): enero, 2011

$\mathrm{O}_{2}$ : Observación $2 \longrightarrow$ postest (encuesta): julio, 2011 
Para la recolección de datos, previas autorizaciones correspondientes, se procedió a aplicar el instrumento antes de la implementación de la estrategia de marketing relacional. El instrumento "Encuesta de fidelización de clientes" consta de 24 declaraciones, estas tienen cinco posibles valores de respuesta según la escala de Likert. Las dimensiones que se determinaron en función del análisis estadístico y cualitativo fueron:

a) Compra

Entendida como la decisión que el cliente realiza luego de haber evaluado entre varias alternativas y obtenido una percepción del producto en términos de calidad, cantidad, variedad, facilidad de adquisición, presentación, disponibilidad de productos, comodidad, duración, entre otros.

b) Servicios

Un servicio es cualquier actividad o beneficio que una parte pueda ofrecer a la otra que sea esencialmente intangible y que no resulte en propiedad de cosa alguna. Su producción puede o no estar ligada a un producto físico. En esta investigación, se entiende el concepto de "servicios" como la atención y facilidades que el cliente percibe que está recibiendo de parte de la organización.

c) Lealtad

La lealtad es un concepto comportamental, al medir la naturaleza de las compras repetitivas a lo largo del tiempo, en sus vertientes de frecuencia (compra recurrente), intención de repetir la compra y recomendarla a otros.

\section{Validez y confiabilidad del instrumento}

A partir de los datos de la muestra piloto se efectuó el análisis de confiabilidad, utilizando el estadístico Alfa de Cronbach, con los resultados siguientes:

Tabla 1

Estadísticos de fiabilidad

\begin{tabular}{cc} 
Alfa de Cronbach & N. ${ }^{\circ}$ de elementos \\
\hline .990 & 24 \\
\hline
\end{tabular}

El coeficiente Alfa de Cronbach es muy cercano a 1.00; por lo tanto, denota una alta confiabilidad. 


\section{Contrastación de Hipótesis}

\section{- Hipótesis General}

La estrategia de marketing relacional mejora la fidelización de los clientes del Centro de Aplicación Productos Unión.

\section{- Forma Estadística}

$\mathrm{H}_{\mathrm{o}}$ : La fidelización antes de aplicar el marketing relacional es igual que despúes de aplicarla.

$\mathrm{H}_{1}$ : La fidelización antes de aplicar el marketing relacional es menor que después de aplicarla.

Tabla 2

Prueba de muestras relacionadas

\begin{tabular}{ccccc} 
& & T & gl & Valor $p$ \\
\hline Par 1 & $\begin{array}{c}\text { Fidelización - Antes } \\
\text { Fidelización - Después }\end{array}$ & -13.731 & 215 & .000 \\
\hline
\end{tabular}

Con el valor obtenido de $\mathrm{p}=0.000$ (menor que 0.05 ) se rechaza $\mathrm{Ho}$, lo cual implica que se acepta nuestra hipótesis. Esto significa que la diferencia entre el nivel de fidelización de los clientes es significativa, a favor de la fidelización "post", con un nivel de confianza del 95\%.

\section{Resultados y discusión}

\subsection{Resultados}

La fidelización de clientes se midió mediante un instrumento organizado en tres dimensiones: Compra, Servicios y Lealtad.

\subsubsection{Dimensión 1: Compra}




\section{Tabla 3}

Dimensión Compra - Fase "ANTES"

\begin{tabular}{|c|c|c|c|}
\hline \multirow{2}{*}{ Afirmación } & \multicolumn{3}{|c|}{ Desviación } \\
\hline & Media & Típica & Observación \\
\hline $\begin{array}{l}\text { Productos Unión me visita de acuerdo a un horario } \\
\text { establecido y siempre que les hago pedidos adicio- } \\
\text { nales (extras). }\end{array}$ & 1.98 & 0.910 & $\begin{array}{l}\text { Medianamente } \\
\text { de acuerdo }\end{array}$ \\
\hline $\begin{array}{l}\text { Productos Unión tiene una gran variedad de produc- } \\
\text { tos y conozco gran parte de ellos. }\end{array}$ & 2.12 & 1.047 & $\begin{array}{l}\text { No lo tiene de- } \\
\text { finido }\end{array}$ \\
\hline $\begin{array}{l}\text { La presentación/imagen de los vehículos, el chofer, } \\
\text { el vendedor, las jabas de Productos Unión es buena. }\end{array}$ & 1.97 & 0.915 & $\begin{array}{l}\text { Medianamente } \\
\text { de acuerdo }\end{array}$ \\
\hline $\begin{array}{l}\text { El precio de los Productos Unión es adecuado, no } \\
\text { tengo problema. }\end{array}$ & 2.35 & 1.123 & $\begin{array}{l}\text { No lo tiene de- } \\
\text { finido }\end{array}$ \\
\hline $\begin{array}{l}\text { Cuando me visita personal de Productos Unión, } \\
\text { se me permite que vea los productos y los pueda } \\
\text { escoger. }\end{array}$ & 1.93 & 0.913 & $\begin{array}{l}\text { Medianamente } \\
\text { de acuerdo }\end{array}$ \\
\hline $\begin{array}{l}\text { En cuanto a calidad, los Productos Unión son mejores } \\
\text { que otros de la competencia. }\end{array}$ & 2.64 & 1.337 & $\begin{array}{l}\text { No lo tiene de- } \\
\text { finido }\end{array}$ \\
\hline $\begin{array}{l}\text { Existe amplio surtido de productos que normalmen- } \\
\text { te pido y me entregan. }\end{array}$ & 2.00 & 0.932 & $\begin{array}{l}\text { Medianamente } \\
\text { de acuerdo }\end{array}$ \\
\hline $\begin{array}{l}\text { Productos Unión me entrega exhibidores y jabas para } \\
\text { mostrar y almacenar lo que compro. }\end{array}$ & 3.14 & 1.279 & $\begin{array}{l}\text { No lo tiene de- } \\
\text { finido }\end{array}$ \\
\hline $\begin{array}{l}\text { El trato que recibo con los vendedores de Productos } \\
\text { Unión es cordial y los considero amigos. }\end{array}$ & 2.08 & 1.012 & $\begin{array}{l}\text { No lo tiene de- } \\
\text { finido }\end{array}$ \\
\hline $\begin{array}{l}\text { El personal de Productos Unión me recibe devolu- } \\
\text { ciones cuando tengo productos sobrantes a punto } \\
\text { de vencer. }\end{array}$ & 1.72 & 0.712 & $\begin{array}{l}\text { Medianamente } \\
\text { de acuerdo }\end{array}$ \\
\hline $\begin{array}{l}\text { Cuando me visitan los vendedores de Productos } \\
\text { Unión recibo alguna sorpresa como obsequios, } \\
\text { incentivos, información sobre los productos, etc. }\end{array}$ & 3.34 & 1.265 & $\begin{array}{l}\text { No lo tiene de- } \\
\text { finido }\end{array}$ \\
\hline $\begin{array}{l}\text { Confío en que los Productos Unión que compro } \\
\text { me van a durar lo suficiente dentro de su fecha de } \\
\text { vencimiento. }\end{array}$ & 2.00 & 0.888 & $\begin{array}{l}\text { Medianamente } \\
\text { de acuerdo }\end{array}$ \\
\hline $\begin{array}{l}\text { No tengo problemas de robo, estafa o algún acto de } \\
\text { mala fe de parte del personal de Productos Unión }\end{array}$ & 1.87 & 0.825 & $\begin{array}{l}\text { Medianamente } \\
\text { de acuerdo }\end{array}$ \\
\hline
\end{tabular}

En el manejo de la dimensión compra en la fase "antes", la muestra se presenta medianamente de acuerdo en los siguientes rubros: atención al 


\section{Juan Carlos Niño de Guzmán Miranda}

cliente cuando este lo requiere, presentación, facilidad para ver los productos, surtido de productos, aceptación de devoluciones, calidad de productos dentro de la fecha de vencimiento y confianza en el personal.

Se manifiesta cómo "no lo tiene definido" en aspectos tales como conocimiento de la variedad de productos, los precios, calidad, facilidades para exhibición de productos, trato con los clientes e incentivos a los compradores.

Tabla 4

Dimensión Compra - Fase "DESPUÉS"

\begin{tabular}{|c|c|c|c|}
\hline \multirow{2}{*}{ Afirmación } & \multicolumn{3}{|c|}{ Desviación } \\
\hline & Media & Típica & Observación \\
\hline $\begin{array}{l}\text { Productos Unión me visita de acuerdo a un horario estable- } \\
\text { cido y siempre que les hago pedidos adicionales (extras). }\end{array}$ & 3.87 & 1.662 & $\begin{array}{l}\text { No lo tiene } \\
\text { definido }\end{array}$ \\
\hline $\begin{array}{l}\text { Productos Unión tiene una gran variedad de productos y } \\
\text { conozco gran parte de ellos. }\end{array}$ & 4.12 & 1.281 & $\begin{array}{l}\text { No lo tiene } \\
\text { definido }\end{array}$ \\
\hline $\begin{array}{l}\text { La presentación/imagen de los vehículos, el chofer, el ven- } \\
\text { dedor, las jabas de Productos Unión es buena. }\end{array}$ & 4.56 & 0.914 & $\begin{array}{l}\text { Medianamen- } \\
\text { te de acuerdo }\end{array}$ \\
\hline $\begin{array}{l}\text { El precio de los Productos Unión es adecuado, no tengo } \\
\text { problema. }\end{array}$ & 4.05 & 1.274 & $\begin{array}{l}\text { No lo tiene } \\
\text { definido }\end{array}$ \\
\hline $\begin{array}{l}\text { Cuando me visita personal de Productos Unión, se me per- } \\
\text { mite que vea los productos y los pueda escoger. }\end{array}$ & 4.23 & 1.262 & $\begin{array}{l}\text { No lo tiene } \\
\text { definido }\end{array}$ \\
\hline $\begin{array}{l}\text { En cuanto a calidad, los Productos Unión son mejores que } \\
\text { otros de la competencia. }\end{array}$ & 4.33 & 0.853 & $\begin{array}{l}\text { Medianamen- } \\
\text { te de acuerdo }\end{array}$ \\
\hline $\begin{array}{l}\text { Existe amplio surtido de productos que normalmente pido } \\
\text { y me entregan. }\end{array}$ & 4.40 & 0.910 & $\begin{array}{l}\text { Medianamen- } \\
\text { te de acuerdo }\end{array}$ \\
\hline $\begin{array}{l}\text { Productos Unión me entrega exhibidores y jabas para mos- } \\
\text { trar y almacenar lo que compro. }\end{array}$ & 2.99 & 1.630 & $\begin{array}{l}\text { No lo tiene } \\
\text { definido }\end{array}$ \\
\hline $\begin{array}{l}\text { El trato que recibo con los vendedores de Productos Unión } \\
\text { es cordial y los considero amigos. }\end{array}$ & 4.75 & 0.796 & $\begin{array}{l}\text { Medianamen- } \\
\text { te de acuerdo }\end{array}$ \\
\hline $\begin{array}{l}\text { El personal de Productos Unión me recibe devoluciones } \\
\text { cuando tengo productos sobrantes a punto de vencer. }\end{array}$ & 4.38 & 1.316 & $\begin{array}{l}\text { No lo tiene } \\
\text { definido }\end{array}$ \\
\hline $\begin{array}{l}\text { Cuando me visitan los vendedores de Productos Unión } \\
\text { recibo alguna sorpresa como obsequios, incentivos, infor- } \\
\text { mación sobre los productos, etc. }\end{array}$ & 1.38 & 0.632 & $\begin{array}{l}\text { Medianamen- } \\
\text { te de acuerdo }\end{array}$ \\
\hline $\begin{array}{l}\text { Confío en que los Productos Unión que compro me van } \\
\text { a durar lo suficiente dentro de su fecha de vencimiento. }\end{array}$ & 4.64 & 0.847 & $\begin{array}{l}\text { Medianamen- } \\
\text { te de acuerdo }\end{array}$ \\
\hline $\begin{array}{l}\text { No tengo problemas de robo, estafa o algún acto de mala } \\
\text { fe de parte del personal de Productos Unión }\end{array}$ & 4.46 & 1.283 & $\begin{array}{l}\text { No lo tiene } \\
\text { definido }\end{array}$ \\
\hline
\end{tabular}


En la fase "después", con respecto a la dimensión compra, la muestra se presenta medianamente de acuerdo en los siguientes rubros: presentación e imagen de productos, calidad, surtido, trato cordial e incentivos por la compra

Mientras tanto, se presenta como "no lo tiene definido" en aspectos tales como disposición para la atención, variedad de productos, precio, facilidades para ver los productos, facilidades para exhibición de productos, recepción de devoluciones y confianza en el personal.

Tabla 5

Dimensión 1-Estadísticos de muestras relacionadas

\begin{tabular}{lcccc} 
& Media & N & Desviación típ. & Coef. Variación \\
\hline Compra Antes & 29.14 & 216 & 12.564 & $43.12 \%$ \\
Compra Después & 47.70 & 216 & 6.292 & $13.19 \%$ \\
\hline
\end{tabular}

Para esta dimensión la media de la valoración de la compra por parte de los clientes, se ha incrementado de 29.14 (antes) a 47.70 (después), pudiéndose observar una disminución de la variabilidad, hecho que se refleja en el coeficiente de variación, que pasa de $43.12 \%$ en la fase antes a $13.19 \%$ en la fase después.

\subsubsection{Dimensión 2: Servicios}

Tabla 6

Dimensión Servicios - Fase "ANTES"

\begin{tabular}{|c|c|c|c|}
\hline \multirow{2}{*}{ Afirmación } & \multicolumn{3}{|c|}{ Desviación } \\
\hline & Media & Típica & Observación \\
\hline $\begin{array}{l}\text { Estoy conforme con la rapidez del servicio cuando hago } \\
\text { mis pedidos. }\end{array}$ & 2.77 & 1.118 & $\begin{array}{l}\text { No lo tiene } \\
\text { definido }\end{array}$ \\
\hline $\begin{array}{l}\text { Los trabajadores de Productos Unión son hábiles y rápidos } \\
\text { en la cobranza y devoluciones }\end{array}$ & 2.58 & 1.273 & $\begin{array}{l}\text { No lo tiene } \\
\text { definido }\end{array}$ \\
\hline $\begin{array}{l}\text { Siempre recibo servicios adicionales de parte de Productos } \\
\text { Unión, como por ejemplo: afiches, orejeras, jaladores, pa- } \\
\text { sacalles, volantes, polos, gorros, promociones, etc. }\end{array}$ & 3.15 & 1.282 & $\begin{array}{l}\text { No lo tiene } \\
\text { definido }\end{array}$ \\
\hline $\begin{array}{l}\text { Los trabajadores de Productos Unión tienen sus productos } \\
\text { bien clasificados y ordenados, tanto físicamente como en } \\
\text { su lista de productos. }\end{array}$ & 2.41 & 1.111 & $\begin{array}{l}\text { No lo tiene } \\
\text { definido }\end{array}$ \\
\hline $\begin{array}{l}\text { Frecuentemente recibo incentivos para ganar premios, } \\
\text { puntos y ofertas de Productos Unión. }\end{array}$ & 3.70 & 1.267 & $\begin{array}{l}\text { No lo tiene } \\
\text { definido }\end{array}$ \\
\hline
\end{tabular}




\section{Juan Carlos Niño de Guzmán Miranda}

\begin{tabular}{lrrr}
$\begin{array}{l}\text { Siempre me dan facilidades de pago cuando compro Pro- } \\
\text { ductos Unión. }\end{array}$ & 1.104 & $\begin{array}{l}\text { No lo tiene } \\
\text { definido }\end{array}$ \\
$\begin{array}{l}\text { Cuando hay campañas (navidad, escolar, etc.), Productos } \\
\text { Unión me da créditos especiales. }\end{array}$ & 1.100 & $\begin{array}{l}\text { No lo tiene } \\
\text { definido }\end{array}$ \\
$\begin{array}{l}\text { Los Productos Unión que compro vienen bien embalados } \\
\text { y no cobran adicionalmente por cajas u otros accesorios. }\end{array}$ & 0.961 & $\begin{array}{l}\text { Medianamente } \\
\text { de acuerdo }\end{array}$ \\
$\begin{array}{l}\text { Siempre tengo la seguridad de recibir los mejores precios } \\
\text { para mi negocio. }\end{array}$ & 2.41 & 1.093 & $\begin{array}{l}\text { No lo tiene } \\
\text { definido }\end{array}$ \\
\hline
\end{tabular}

En la fase "antes" de la dimensión Servicios, se cataloga como medianamente de acuerdo al ítem que tiene relación con el embalaje y facilidades de manipulación de los productos.

Entre tanto en la misma fase "antes", no lo tienen definido en los rubros de: rapidez del servicio, cobranza y devoluciones oportunas, servicios adicionales, clasificación, incentivos y premios para el cliente, facilidades de pago, créditos y precios.

Tabla 7

Dimensión Servicios - Fase "DESPUÉS"

\begin{tabular}{llcl}
\hline \multicolumn{1}{c}{ Afirmación } & \multicolumn{2}{c}{ Desviación } \\
\cline { 2 - 4 } & Media & Típica & Observación \\
\hline $\begin{array}{l}\text { Estoy conforme con la rapidez del servicio cuando hago } \\
\text { mis pedidos. }\end{array}$ & 4.66 & 0.819 & $\begin{array}{l}\text { Medianamente } \\
\text { de acuerdo }\end{array}$ \\
$\begin{array}{l}\text { Los trabajadores de Productos Unión son hábiles y rápidos } \\
\text { en la cobranza y devoluciones }\end{array}$ & 0.64 & 0.746 & $\begin{array}{l}\text { Medianamente } \\
\text { de acuerdo }\end{array}$ \\
$\begin{array}{l}\text { Siempre recibo servicios adicionales de parte de Productos } \\
\text { Unión, como por ejemplo: afiches, orejeras, jaladores, pasa- }\end{array}$ & 1.540 & $\begin{array}{l}\text { No lo tiene } \\
\text { definido }\end{array}$ \\
$\begin{array}{l}\text { calles, volantes, polos, gorros, promociones, etc. } \\
\begin{array}{l}\text { Los trabajadores de Productos Unión tienen sus productos } \\
\text { bien clasificados y ordenados, tanto físicamente como en } \\
\text { su lista de productos. }\end{array}\end{array}$ & 0.621 & $\begin{array}{l}\text { Medianamente } \\
\text { de acuerdo }\end{array}$ \\
$\begin{array}{l}\text { Frecuentemente recibo incentivos para ganar premios, } \\
\text { puntos y ofertas de Productos Unión. }\end{array}$ & 1.33 & 0.877 & $\begin{array}{l}\text { Medianamente } \\
\text { de acuerdo }\end{array}$ \\
$\begin{array}{l}\text { Siempre me dan facilidades de pago cuando compro Pro- } \\
\text { ductos Unión. }\end{array}$ & 2.83 & 1.621 & $\begin{array}{l}\text { No lo tiene } \\
\text { definido }\end{array}$ \\
$\begin{array}{l}\text { Cuando hay campañas (navidad, escolar, etc.), Productos } \\
\text { Unión me da créditos especiales. }\end{array}$ & 1.90 & 1.360 & $\begin{array}{l}\text { No lo tiene } \\
\text { definido }\end{array}$ \\
$\begin{array}{l}\text { Los Productos Unión que compro vienen bien embalados } \\
\text { y no cobran adicionalmente por cajas u otros accesorios. }\end{array}$ & 4.56 & 1.076 & $\begin{array}{l}\text { No lo tiene } \\
\text { definido }\end{array}$ \\
$\begin{array}{l}\text { Siempre tengo la seguridad de recibir los mejores precios } \\
\text { para mi negocio. }\end{array}$ & 4.45 & 1.048 & $\begin{array}{l}\text { No lo tiene } \\
\text { definido }\end{array}$ \\
\hline
\end{tabular}


Se muestra en esta fase que los rubros identificados como medianamente de acuerdo son: rapidez, cobranza, clasificación e incentivos para ganar premios.

No lo tienen definido en la fase "después" las afirmaciones de: servicios adicionales, facilidades de pago, créditos especiales, cobro por embalajes y seguridad de recibir mejores precios.

Tabla 8

Dimensión Estadísticos de muestras relacionadas

\begin{tabular}{lcccc} 
& Media & N & $\begin{array}{c}\text { Desviación } \\
\text { Típica }\end{array}$ & Coef. Variación \\
\hline Servicios Antes & 25.96 & 216 & 9.679 & $37.28 \%$ \\
Servicios Después & 31.53 & 216 & 3.230 & $10.24 \%$ \\
\hline
\end{tabular}

La media de la valoración de los servicios por parte de los clientes se ha incrementado de 25.96 (antes) a 31.53 (después), pudiéndose observar una disminución de la variabilidad, hecho que se refleja en el coeficiente de variación, que pasa de $37.28 \%$ en la fase antes a $10.24 \%$ en la fase después.

\subsubsection{Dimensión 3: Lealtad}

Tabla 9

Dimensión - Lealtad - Fase "ANTES"

\begin{tabular}{llcc}
\hline \multicolumn{1}{c}{ Afirmación } & \multicolumn{3}{c}{ Desviación } \\
\cline { 2 - 4 } & Media & Típica & Observación \\
\hline $\begin{array}{l}\text { Compro Productos Unión porque siempre lo hice, mis clien- } \\
\text { tes también lo piden. }\end{array}$ & 2.02 & 0.791 & $\begin{array}{l}\text { Medianamente } \\
\text { de acuerdo }\end{array}$ \\
$\begin{array}{l}\text { Siempre que me piden algún producto saludable, compro } \\
\text { Productos Unión y recomiendo su consumo. }\end{array}$ & 1.76 & 0.754 & $\begin{array}{l}\text { Medianamente } \\
\text { de acuerdo }\end{array}$ \\
\hline
\end{tabular}

En la fase "antes", la dimensión Lealtad, la muestra valoró como medianamente de acuerdo a los ítems de compra de Productos Unión porque siempre lo hicieron y sus clientes también lo piden; y recomendación de consumo de Productos Unión porque son productos saludables. 
Tabla 10

Dimensión Lealtad - Fase "DESPUÉS"

\begin{tabular}{llll}
\hline \multicolumn{1}{c}{ Afirmación } & \multicolumn{3}{c}{ Desviación } \\
\cline { 2 - 4 } & Media & Típica & Observación \\
\hline $\begin{array}{l}\text { Compro Productos Unión porque siempre lo hice, mis clien- } \\
\text { tes también lo piden. }\end{array}$ & 4.83 & 0.570 & $\begin{array}{l}\text { Medianamente } \\
\text { de acuerdo }\end{array}$ \\
$\begin{array}{l}\text { Siempre que me piden algún producto saludable, compro } \\
\text { Productos Unión y recomiendo su consumo. }\end{array}$ & 4.66 & 0.768 & $\begin{array}{l}\text { Medianamente } \\
\text { de acuerdo }\end{array}$ \\
\hline
\end{tabular}

En la fase "después", la dimensión Lealtad, se encontró que también califican como medianamente de acuerdo a los ítems de de compra de Productos Unión porque siempre lo hicieron y sus clientes también lo piden; y recomendación de consumo de Productos Unión porque son productos saludables, pero esta vez con una media mucho mayor.

Tabla 11

Dimensión 3- Estadísticos de muestras relacionadas

\begin{tabular}{lcccc} 
& Media & N & $\begin{array}{c}\text { Desviación } \\
\text { Típica }\end{array}$ & Coef. Variación \\
\hline Lealtad Antes & 3.78 & 216 & 1.481 & $39.18 \%$ \\
Lealtad Después & 9.49 & 216 & 0.959 & $10.11 \%$ \\
\hline
\end{tabular}

Para la dimensión Lealtad se observa que la media de la valoración se ha incrementado de 3.78 (antes) a 9.49 (después), pudiéndose observar una disminución de la variabilidad, hecho que se refleja en el coeficiente de variación, que pasa de $39.18 \%$ en la fase antes a $10.11 \%$ en la fase después.

\subsubsection{Fidelización del Cliente}

Tabla 12

Fidelización de Clientes: Estadísticos de muestras relacionadas

\begin{tabular}{lcccc} 
& Media & N & $\begin{array}{c}\text { Desviación } \\
\text { Típica }\end{array}$ & Coef. Variación \\
\hline Fidelización - Antes & 59.82 & 216 & 22.96 & $38 \%$ \\
Fidelización - Después & 93.25 & 216 & 8.46 & $9 \%$ \\
\hline
\end{tabular}


Considerando el total de la prueba aplicada se ha obtenido un incremento del valor promedio de la fidelización de los clientes de 59.82 (antes) a 93.25 (después). Observándose también que el coeficiente de variación se ha reducido de $38 \%$ a $9 \%$, tornándose más homogéneo en la fase "después".

\subsection{Discusión}

La economía actual en todo el mundo está siendo dominada por los servicios (Duran, 2008) y en la medida que va en aumento, las organizaciones han visto conveniente elaborar estrategias para ceñirse a este contexto. El marketing es una de las herramientas más indicadas para competir adecuadamente. Tradicionalmente, el marketing ha estado enfocado en lo transaccional, por ello que se priorizaba conseguir clientes para efectuar la venta y así terminaba la relación con ellos.

No obstante, el marketing relacional, se ha ocupado de esta falencia asignándole mayor importancia a captar, convencer y conservar clientes con el objetivo de fidelizarlos. Es así que Grönroos (1997), propone que a lo largo de un eje podrían representarse las diferentes aproximaciones estratégicas que una empresa podría adoptar en cuanto a sus relaciones. En un extremo de este eje se encontraría el marketing relacional cuyo fondo de atención se centra en la construcción y en el mantenimiento de relaciones. En el otro extremo del eje se encontraría el marketing transaccional, donde el énfasis está puesto en cada una de las transacciones independientes que se realizan.

Coincidentemente, en el extremo del marketing transaccional encajan las empresas que se dedican a la fabricación o confección de bienes y en el otro extremo del marketing relacional se encuentran las empresas que brindan servicios.

En este sentido, muchas empresas de producción tienen que poner atención al tema de servicios, por ende al marketing relacional, puesto que los clientes desean ser reconocidos individualmente, atendidos de manera especial y constantemente comunicados acerca de cualquier novedad de su marca preferida. Al respecto, Grönroos (1996) y Webster (1994) dan sustento a ello mencionando que cualquier empresa que adopta una estrategia relacional se convierte en una empresa de servicios.

En el caso del Centro de Aplicación Productos Unión, tradicionalmente orientada a la elaboración de productos alimenticios, la inserción de la estrategia de marketing relacional es decisiva para obtener clientes más fieles.

Los resultados hallados así lo demuestran, puesto que en las dimen- 
siones de compra, servicios y lealtad, se puede constatar un incremento de la percepción de cada uno de estos conceptos luego de haberse implementado la estrategia de marketing relacional.

Muchos estudios demuestran que la aplicación de una estrategia de marketing relacional brinda beneficios a todas las partes implicadas en la red que se establece. Las empresas del sector servicios son las que obtienen una mayor rentabilidad de la aplicación de una estrategia de este tipo. Por ejemplo, según una investigación realizada por Sin, ét al. (2002) las empresas de servicios que adoptan una orientación de marketing relacional consiguen mejorar su rendimiento, tanto por lo que se refiere a los resultados de marketing, representados por indicadores como "crecimiento de las ventas; cuota de mercado; retención de clientes", como por lo que se refiere a resultados financieros, "rentabilidad de la inversión".

El primer beneficio que se puede obtener es la reducción de la tasa de pérdida de clientes. El segundo, se refiere a que un cliente genera más beneficios para la empresa, a medida que la relación entre ambos se hace más duradera. El tercer beneficio, que los clientes satisfechos se convierten habitualmente en recomendadores del producto o del servicio que ofrece la empresa (Reichheld y Sasser, 1990).

En general, lo que obtienen los clientes con la aplicación de la estrategia de markerting relacional son un mayor valor agregado, una mejor calidad de servicio basada en un trato personalizado, una disminución de los costos de transacción y, por ende, un incremento de la satisfacción (Iglesias, citado por Alfaro, 2004).

Por otro lado, el estudio que se realizó fue dirigido a una muestra de bodegueros -intermediarios que llegan a tener contacto con el cliente final-. Concerniente a esto, Grönroos (1997), menciona que la gestión de las relaciones con los distribuidores es un factor clave para las empresas que elaboran productos de consumo masivo. Esta situación ha promovido la generación de áreas de trade marketing cuya función es gestionar estas relaciones. Utilizamos este sustento teórico para el caso del Centro de Productos Unión, puesto que es factible su aplicación, dada la naturaleza del mismo.

Se ha encontrado que las actividades correspondientes al mix de marketing (mezcla comercial) no se ven opacadas ni tienen puntos en contra de la aplicación paralela de la estrategia de marketing relacional y se coincide con Alfaro (2004), quien también menciona que el marketing relacional puede aplicarse de manera conjunta con el mix de marketing. 


\section{Conclusiones}

1. Se notó una mejora en la fidelización de los clientes del Centro de Aplicación Productos Unión del período inicial hasta haberse aplicado la estrategia de marketing relacional y medir su efectividad seis meses después. Con esto se puede sustentar claramente la hipótesis de que la estrategia de marketing relacional mejora la fidelización de los clientes. Es posible observar esta mejora con datos descriptivos donde el incremento de las medias fueron 59.82 (antes) a 93.25 (después) según la Tabla $N^{\circ} 12$ y con el coeficiente de variación reducido de $38 \%$ a $9 \%$, siendo más homogéneo en la fase después. Asimismo, la prueba t-student con el valor de $p=0.000$ demuestra que estadísticamente hubo una mejora muy significativa en la fidelización de los clientes luego de haberse aplicado la estrategia de marketing relacional.

2. Se notó una mejora significativa en la compra de los clientes del Centro de Aplicación Productos Unión comparado desde el período inicial hasta haberse aplicado la estrategia de marketing relacional y posteriormente medir su efectividad seis meses después. De manera descriptiva es posible observar esta mejora donde el incremento de las medias fue de 29.14 (antes) a 47.70 (después) según la Tabla $N^{\circ} 5$, con coeficiente de variación reducida de $43 \%$ a $13 \%$, tornándose así más homogéneo en la fase "después". Asimismo, la prueba t-student con el valor de $p=0.000$ demuestra que estadísticamente hubo una mejora muy significativa en la compra de los clientes luego de haberse aplicado la estrategia de marketing relacional.

3. La percepción de los clientes en cuanto a los servicios que reciben del Centro de Aplicación Productos Unión mejoró luego de haberse aplicado la estrategia de marketing relacional. Es posible observar esta mejora de manera descriptiva donde el incremento de las medias fueron de 25.96 (antes) a 31.53 (después) según la Tabla $N^{\circ} 8$, con variabilidad reducida que se demuestra en el coeficiente de variación que pasa de $37 \%$ a $10 \%$ en la fase "después". Asimismo, la prueba t-student con el valor de $p=0.000$ demuestra que estadísticamente hubo una mejora muy significativa en la percepción de los servicios que reciben los clientes luego de haberse aplicado la estrategia de marketing relacional. 
4. La lealtad de los clientes del Centro de Aplicación Productos Unión mejoró luego de haberse efectuado la estrategia de marketing relacional. De manera descriptiva es posible observar esta mejora donde el incremento de las medias fueron de 3.78 (antes) a 9.49 (después) según la Tabla $N^{\circ} 11$, con coeficiente de variación reducido de $39 \%$ a $10 \%$ que demuestra que en la fase después se tornó más homogéneo. Asimismo, la prueba t-student con el valor de $\mathrm{p}=0.000$ demuestra que estadísticamente hubo una mejora muy significativa en la lealtad de los clientes luego de haberse aplicado la estrategia de marketing relacional.

\section{Recomendaciones}

Habiendo encontrado relación significativa entre la estrategia de marketing relacional y la fidelización de los clientes, hacemos las siguientes recomendaciones:

1. Realizar investigaciones a mayor escala para efectos de poder aplicar la estrategia en organizaciones de diversos sectores económicos.

2. Realizar investigaciones relacionadas al conocimiento más exhaustivo de los conceptos de comportamiento de compra y percepción del servicio con el fin de diseñar nuevas estrategias para llegar a sobrepasar expectativas de los clientes.

3. El Centro de Aplicación Productos Unión puede dar un impulso mayor a esta estrategia para obtener mejores resultados en los diferentes canales de venta que posee, esto sería útil para transferir el conocimiento desarrollado a otras organizaciones que forman parte de su corporación.

4. Se debe fomentar la aplicación de marketing relacional en diferentes sectores de la sociedad para que puedan llegar a obtener un mayor número de clientes fieles.

5. Velar por el mantenimiento de la cartera de clientes valiosos y su constante actualización debido a que la información contenida es un activo intangible invalorable. Con ella se pueden multiplicar tácticas y actividades con el fin de fidelizar clientes para la organización. 
6. Las organizaciones que implementan la estrategia de marketing relacional deben tener muy en cuenta la importancia que demanda el marketing interno, que tiene que ver con la aplicación del marketing para el interior de la organización. Para ello, se deberá indagar acerca de las necesidades, en orden de prioridad, que poseen los trabajadores, los elementos motivadores, descubrir a los trabajadores que generan valor agregado dentro de la cadena de valor de la organización, mantener una fluida comunicación con ellos -formal e informal-y retener a los trabajadores realmente valiosos. Se sugiere, por lo tanto, ampliar el conocimiento de la aplicación del marketing interno para tener un equipo de trabajo identificado y comprometido con la organización, orientado a la satisfacción plena del cliente y que llegue a cumplir los objetivos trazados. De esta manera, con clientes internos satisfechos se podrá obtener clientes externos también satisfechos.

Juan Carlos Niño de Guzmán

Universidad Peruana Unión

e-mail: jcnino@upeu.edu.pe

Recibido: 15 de mayo de 2014 Aceptado: 20 de julio de 2014 
Juan Carlos Niño de Guzmán Miranda

\section{Referencias}

Acevedo, A. (2008). Tesis: "Modelo de gestión de las relaciones con los clientes para clusters de pymes peruanas del sector de confecciones para la exportación". UNMSM.

Alfaro, M. (2004). Temas clave en marketing relacional. España. Madrid, España: McGraw-Hill / Interamericana.

Alfaro, Manuel. (2004). Temas clave en marketing relacional. Mcgraw-Hill / Interamericana de España. Madrid, España.

Bernal, C. (2010). Metodología de la Investigación. Pearson Educación. Colombia.

Durán, J. (2008). El Comercio de Servicios en los países de América Latina y el Caribe: Evaluación Cuantitativa y normativa. Santiago, Chile 11-14, Noviembre.

Grönroos, C. (1996). Relationship Marketing: Strategic and tactical implications. Journal of Management Decision. N 34. Pp. 5-14.

Grönroos, C. (1997). From marketing mix to relationship marketing - towards a paradigm shift in marketing. Journal of Management Decision. 35 (4): 322-229.

Mesquita, J., y Lara, J. (2007). Atributos determinantes da lealdade á loja: estudo do setor supermercadista. Brazilian Business Review. 4. (3). p. 233-251.

Payne, C.; Ballantyne, D. y Martin, C. (2005). A stakeholder approach to relationship marketing strategy: The development and us of the 'six markets' model". European Journal of Marketing. Vol. 39 Iss: 7/8, pp. $855-871$.

Reichheld, F. (2002). The loyalty effect. The hidden force behind growth, profits ad last value. Boston, Harvard School Press./ Versión en español, Ariel Empresa.

Sheth, J.N.; Mittal, B. Y Newman, B.I. (2001). Comportamento do cliente: Indo além do comportamento do consumidor. Sao Paulo: Atlas.

Sin, L.Y.M.; Tse, A.C.B.; Yau, O.H.M.; Lee, J.S.Y. Y Chow, r. (2002). The effect of relationship marketing orientation on business performance in service-oriented economy. Journal of Services Marketing. $\mathrm{N}^{\circ}$ 16(7): pp. 656-676.

Volle, P., y Mimouni-Chaabane, A. (2010). Perceived benefits of loyalty programs: Scale development and implications for relational strategies. Journal of Business Research, vol. 63. (1). pp. 32-37.

Webster, F.E. (1994). Executing the new marketing concept. Journal of Marketing Management. $\mathrm{N}^{\circ} 3, \mathrm{pp} .9-16$. 